\title{
Erratum: Effect of Aldosterone on the Regulation of the Volume of Principal Cells of Rat Cortical Collecting Duct Epithelium in Early Postnatal Development [Doklady Biological Sciences 423 (6), 385-388 (2008)]
} N. S. Logvinenko, E. I. Solenov, and Academician L. N. Ivanova

DOI: $10.1134 / \mathrm{S} 001249660902032 \mathrm{X}$

N. S. Logvinenko, E. I. Solenov, and Academician L. N. Ivanova acknowledge the partial support for their study on "Effect of Aldosterone on the Regulation of

Institute of Cytology and Genetics, Siberian Branch, Russian Academy of Sciences, pr. Akademika Lavrent'eva 10, Novosibirsk, 630090 Russia the Volume of Principal Cells of Rat Cortical Collecting Duct Epithelium in Early Postnatal Development" published in Doklady Biological Sciences, 2008, vol. 423, pp. 385-388 from the Russian Foundation for Basic Research (project nos. 08-04-00658-a and 08-04-00541-a) and Program for Support of Leading Scientific Schools (project no. NSh-7071.2006.4). 